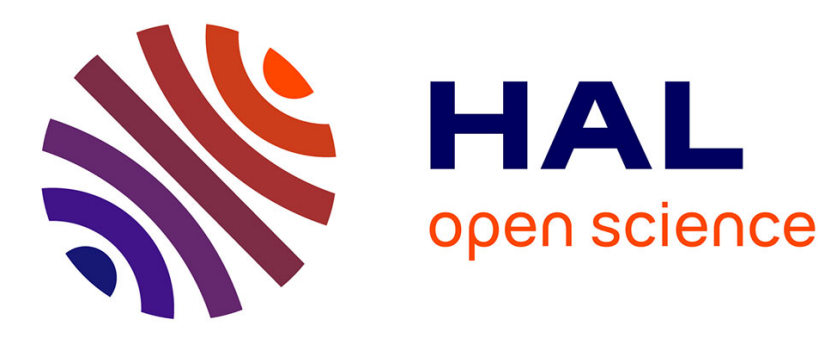

\title{
Kinetic study of the mirror mode
}

Vincent Génot, S. J. Schwartz, C. Mazelle, M. Balikhin, M. Dunlop, T. M.

Bauer

\section{To cite this version:}

Vincent Génot, S. J. Schwartz, C. Mazelle, M. Balikhin, M. Dunlop, et al.. Kinetic study of the mirror mode. Journal of Geophysical Research, 2001, 106, pp.21611-21622. 10.1029/2000JA000457 . hal-00290083

\section{HAL Id: hal-00290083 https://hal.science/hal-00290083}

Submitted on 25 Jun 2008

HAL is a multi-disciplinary open access archive for the deposit and dissemination of scientific research documents, whether they are published or not. The documents may come from teaching and research institutions in France or abroad, or from public or private research centers.
L'archive ouverte pluridisciplinaire HAL, est destinée au dépôt et à la diffusion de documents scientifiques de niveau recherche, publiés ou non, émanant des établissements d'enseignement et de recherche français ou étrangers, des laboratoires publics ou privés. 


\title{
Kinetic study of the mirror mode
}

\author{
V. Génot ${ }^{1}$, S. J. Schwartz ${ }^{1}$, C. Mazelle ${ }^{2}$, M. Balikhin ${ }^{3}$, M. Dunlop ${ }^{4}$, and T. \\ M. Bauer ${ }^{5}$
}

Abstract. The linear Vlasov dispersion is solved to reveal the kinetic properties of the mirror mode. The existence of the torsional component of both the magnetic and velocity perturbations are kinetic features that are not explained by MHD theory. A parameter study is then employed to clarify the behavior of these particular components under different plasma conditions. The key parameters are the ion temperature anisotropy and the plasma to magnetic pressure ratio which act in similar ways to control the value of the torsional (or Alfvénic) components. Data from the Active Magnetospheric Particle Tracer Explorers mission show that under most magnetosheath plasma conditions the magnetic torsional component is negligible, whereas the velocity one is always larger or comparable to the other components. This experimental illustration also shows that there exist elliptically polarized mirror modes, contrary to what our, and all previous, analytical treatments predict.

\section{Introduction}

Mirror waves have been encountered in many different plasma environments such as the magnetosheath of the Earth [Denton et al., 1995], the solar wind [Winterhalter et al., 1994], the surroundings of comet Halley [Russel et al., 1987; Glassmeier et al., 1993] or those of Io [Huddleston et al., 1999]. This ubiquity demands continued investigation to identify and characterize the mode, its growth, and its consequences.

These waves are nonpropagating (zero real frequency in the plasma frame of reference) and typically display an anticorrelation between the plasma and magnetic field perturbations. This last feature is also one of the slow mode, often confused with the mirror mode. Mirror modes can be generated by the mirror instability that takes place in high- $\beta$ plasmas with temperature anisotropies in one or several species. Although the mirror instability is often considered a fluid instability, it has been shown that it cannot be treated in the fluid approximation [Southwood and Kivelson, 1993]. Nevertheless, Belmont et al. [1992] have shown that the kinetic calculations can be done in a fluid-like way which simplifies the analytic treatment [see Belmont and Mazelle, 1992]; the mirror mode is then identified as a proper mode and, unlike the MHD treatments, is distinct from the slow mode. From these works it is thus clear that the MHD approach is potentially misleading. Although it is possible to recover the anticorrelation between the plasma and magnetic field perturbations with an MHD treatment, some obvious features are ig- 
nored which lead to inconsistency as we shall see in the following.

From both simulation and experimental points of view, the question of the mirror mode identification has been addressed for some time. Different methods have been established (transport ratios, wave propagation characteristics), none of them being completely satisfactory. This issue has been tackled using different theoretical frameworks, based either on MHD theory [Song et al., 1994; Denton et al., 1995] or linear kinetic theory [Lacombe et al., 1992; Lacombe and Belmont, 1995; Hubert et al., 1998]. The comparison between the theoretical properties of both bifluid MHD and kinetic theories for low-frequency waves has been addressed by [Krauss-Varban et al., 1994]. Recently, Lin et al. [1998] proposed a new method based on the $90^{\circ}$ phase difference between $\delta \vec{B}$ and $\delta \vec{v}$, the magnetic field and the plasma velocity fluctuations, which exists for the mirror mode only. More precisely the phase difference lies between $\delta \vec{B}$ and $\left(\delta \vec{v} \times \vec{B}_{0}\right) \times \vec{k}(\vec{k}$ is the wave vector and $\vec{B}_{0}$ is the ambient magnetic field). This method was illustrated using experimental data (from Active Magnetospheric Particle Tracer Explorers (AMPTE) mission), and theoretical studies performed by the authors (MHD) as well as by Gary [1992] (kinetic theory) and McKean et al. [1992] (hybrid simulations). In the discussion, Lin et al. [1998] recall that MHD analysis does not allow magnetic and velocity components out of the plane $\left(\vec{k}, \vec{B}_{0}\right)$, the so-called coplanarity plane, except for the propagating Alfvén wave. However, their conclusion relies heavily on the existence of this velocity component in the mirror mode which is actually found to be large, if not the largest, both in experimental and simulation data. They used this component to check the $90^{\circ}$ phase difference with the component of the magnetic field fluctuations in the plane $\left(\vec{k}, \vec{B}_{0}\right)$. Although the results of Lin et al. [1998] are worthy of attention (as they are consistent with simulations and experiments), it is essential to understand the origin and behavior of the noncoplanar components: from the experimental point of view, the existence of this velocity component can help to identify the mirror mode. From the theoretical point of view, it highlights the inappropriateness of MHD which cannot explain it. An explanation of this difference is proposed based on the work of Southwood and Kivelson [1993].

From a theoretical point of view the criterion that leads to the triggering of the instability is, in the most common case where only the protons are anisotropic,

$$
A_{p} \geq 1+1 / \beta_{\perp p}
$$


where $A_{p}=T_{\perp p} / T_{\| p}$ is the proton anisotropy and $\beta_{\perp p}=2 \mu_{0} n k T_{\perp p} / B_{0}^{2} . T_{\perp p}$ and $T_{\| p}$ are the ion temperature perpendicular and parallel to the magnetic field, respectively; $n$ is the density. Similar quantities are defined for the electron population. Equation (1) states that the instability criterion is favored in high- $\beta$ plasmas. Increasing anisotropy acts in the same way, but for too large an anisotropy and for a two-species plasma, the ion cyclotron mode develops driven by the free energy contained in the anisotropy (its growth rate overcomes that of the mirror mode, for different propagation angle, though). The mirror mode is therefore prevalent in high- $\beta$, low-anisotropy plasmas. The growth rate of both the mirror and the firehose instability (in the case $\beta_{\| p}=1=A_{e}$ which we consider further below) is given by the following expression, depending on the angle of propagation. The mirror mode appears at large angle (or $k_{\|} \ll k_{\perp}$ ) and the firehose mode at small angle (or $k_{\|} \gg k_{\perp}$ ) [e.g., Hasegawa, 1975]:

$$
\frac{\gamma}{k_{\|} V_{t h \|}}\left(\frac{\pi}{2}\right)^{\frac{1}{2}}=1-\frac{1}{A_{p}}-\frac{1}{A_{p}^{2}}-\frac{k_{\|}^{2}}{2 k_{\perp}^{2}}\left(\frac{1}{A_{p}}-\frac{1}{A_{p}^{2}}\right)
$$

where $V_{t h \|}=\sqrt{2 k T_{\| p} / m_{p}}$ is the parallel proton thermal velocity. Although we are only concerned in the mirror mode case, we shall use this complete expression in a following section. Equation (2) shows that the mirror mode grows with the anisotropy as expected. This equation also shows that, for a given anisotropy and propagation angle, the growth rate is a linear function of the wave number as long as the finite Larmor radius $\left(\rho_{i}\right)$ effects are negligible (Equation $(2)$ is obtained assuming $\left.k \rho_{i} \ll 1\right)$.

In this paper, in light of previous work and in order to search for the origin of the torsional (or Alfvénic) component of magnetic and/or velocity perturbations, we investigate the kinetic properties of the mirror mode. We demonstrate that these components do indeed exist for simple, small-amplitude fluctuations. They are pure kinetic (hot plasma) features which cannot be revealed by a MHD treatment. We establish the range of parameters where the torsional magnetic perturbation can definitely not be neglected. As far as the velocity perturbations are concerned, we show that the component in the Alfvénic direction can be the dominant one. The result of this analysis leads us to point out that, although linearly polarized, the magnetic perturbation linked to the kinetic mirror mode does not lie in the coplanarity plane $\left(\vec{k}, \vec{B}_{0}\right)$ but can be tilted depending on the value of the temperature anisotropy. Therefore the coplanarity theorem cannot be used for the kinetic 
mirror mode. The results concerning the mirror mode growth as function of $\beta_{\|}$and $A_{p}$, in section 3 , confirm those found by Gary [1992]. Section 2 briefly presents the general frame of reference in which the analysis will be conducted, whereas section 4 is devoted to the experimental analysis performed on AMPTE data. We find surprising deviations from even the kinetic properties expected from earlier sections. In the conclusion we discuss the implications of these results.

\section{An Adequate Approach: The Kinetic Theory}

The existence of the noncoplanar component of the magnetic perturbation has previously been demonstrated by Hubert et al. [1998]. Using the program Waves in Homogeneous, Anisotropic Multicomponent Plasmas (WHAMP) [Rönnmark, 1982], these authors calculated the variations with $\beta_{p}$ of the angle between the WHAMP computed wave vector and the one defined by the coplanarity theorem for the mirror mode. In that paper they followed a method initiated by Fazakerkey and Southwood [1994], who investigated the threedimensional (3-D) structure of the mirror mode: in an attempt to depart from the planar wave model, they estimated the spatial dimensions of the mirror structures. However, in the present paper, because our final goal is to compare observations with linear kinetic theory, we shall confine ourselves to the more limited planar wave model (single wave vector).

The coordinate system we choose is such that the ambient magnetic field $\vec{B}_{0}$ is along $\hat{z}$. The wave vector $\vec{k}$ is in the plane $(\hat{x}, \hat{z})$, the coplanarity plane, and $\theta_{k B}$ is the angle between $\vec{B}_{0}(\hat{z})$ and $\vec{k}$. The $\hat{y}$ direction is termed the Alfvénic or torsional direction, perpendicular to the coplanarity plane. From MHD analysis one finds that the mirror mode oscillations are in the coplanarity plane and perpendicular to $\vec{k}\left(\delta B_{k}=\delta B_{y}=0\right)$; we call this direction $\hat{\xi}$; the triad $(\hat{\xi}, \hat{y}, \hat{k})$ is orthonormal (with $\hat{k}=\vec{k} / k$ ). From kinetic theory we shall show that the mirror wave perturbation is in fact of the form: $\delta \vec{B}_{\text {wave }}=\delta B_{\xi} \hat{\xi}+\delta B_{y} \hat{y}$. This is sketched in Figure 1 .

MHD theory strictly prohibits components perpendicular to the coplanarity plane, apart from those associated with pure Alfvén waves. However, both observations and simulations show that $\delta B_{y} \neq 0$ and $\delta v_{y} \neq 0$ in mirror mode episodes. Therefore we have considered the kinetic aspect of this mode and solved the linear Vlasov dispersion relation. To achieve this goal, we use the program WHAMP to perform a parametric study of the linear Vlasov dispersion relation. Indeed, 
we shall use a modified version of this program (implemented by G. Belmont, C. Lacombe, L. Rezeau, and V. Robert) which includes calculations of the velocity perturbations. The results are sensitive functions of both ion and electron anisotropies and of the pressure ratio $\beta$. They are presented in the following section. In order to check that our results are effectively bound to kinetic theory and are not a bifluid effect, we conducted a test for which the electron mass was alternatively enhanced and decreased: the mirror mode characteristics were very slightly modified in both cases. This rules out potential explanations of the results based on the difference of mass of the particles.

\section{Parametric Study}

\subsection{Effects of the Ion Anisotropy and of the Pressure Ratio}

In this section we consider no electron temperature anisotropy; their temperature $T_{e}$ is set such that $T_{e} / T_{\| p}=$ 0.01 , in order to minimize their influence and focus on ion effects only (see Pantellini and Schwartz [1995] and Pokhotelov et al. [2000] for the modifications to some of the mode properties for warm and anisotropic electrons respectively, as well as section 3.2). The magnetic field magnitude $B_{0}$ and the density $n$ are constant. We first investigate here the effect of the proton temperature anisotropy $A_{p}$ on the polarization of the wave $\left(P=i \delta B_{\xi} / \delta B_{y}\right)$, the wavelength $(2 \pi / k)$, the growth rate $\left(\gamma\right.$, for waves varying as $\left.e^{-i \omega t+\gamma t}\right)$, the propagation angle $\left(\theta_{k B}\right)$, and finally the Alfvénic component $\left(\delta B_{y}\right)$. The anisotropy varies from 1.8 to 6 , and the different panels given in the first column of Figure 2 are at maximum growth. We also choose $\beta_{\| p}=1$. Figure 2a gives important information about a kinetic property of the mirror mode: the ratio $P=i \delta B_{\xi} / \delta B_{y}$ is purely imaginary, which means that $\delta B_{\xi} / \delta B_{y}$ is a real constant and thus the mode is linearly polarized. The absolute value of this ratio goes from high values for small anisotropy to values $\leq 10$ for an anisotropy greater than 3 . Above this anisotropy the Alfvénic component is no longer negligible as it exceeds $10 \%$ of the wave amplitude (see Figure 2c).

At this stage we would like to comment on the relation between the linear polarization of the mirror mode and the fact that it is a purely growing mode. In this case $(\omega=i \gamma)$, the properties of the Fried and Conte [1961] function together with Barnes's [1968] results in the case of bi-Maxwellian distribution functions reveal that the dielectric tensor is purely real. It follows that the components of the fluctuating electric field are in 
phase with one another. The same holds, via Faraday's law, for the magnetic components. Hence purely growing modes such as the mirror mode are always linearly polarized. It follows that departures from linear polarization (more adequately termed ellipticity) of a wave mode implies $\omega \neq 0$, that is we deduce that an elliptically polarized mode cannot be purely growing. Therefore, theoretically, an observed elliptic mode cannot be a mirror mode; we shall return to this apparently trivial remark in next section.

The behavior of $\gamma$ shown in Figure $2 \mathrm{~b}$ is the same as given by Gary [1992]: it is basically a growing function of $A_{p}$ with no finite limit. It should not be mistaken with the one given by (2) which yields, in the $k_{\|} \ll k_{\perp}$ ) limit,

$$
\gamma \propto 1-\frac{1}{A_{p}}-\frac{1}{A_{p}^{2}},
$$

which is a function bounded by 1 . Indeed, Figure $2 \mathrm{~b}$ shows the maximum value of $\gamma$ as a function of $A_{p}$ but for the actual values of $\left(\theta_{k B}, k\right)$, different for each $A_{p}$, which lead to this maximum. From the same plot we also see that for increasing anisotropies the maximum growth rate appears at smaller and smaller wavelength. This behavior could be expected from (2), but once again attention must be paid as wave number is plotted here for varying anisotropy and propagation angle.

The propagation angle is also a quantity of interest regarding the wave mode (although the term "propagation" is not pertinent for the mirror mode for which $\omega=0$ ). The basic trend of variation (see Figure 2c) is that the increase of the ion anisotropy leads to a greater departure from the marginal stability condition and allows propagation, which near marginal stability is essentially perpendicular, to move to quasi-perpendicular near $\theta_{k B} \simeq 50^{\circ}$. This can be understood from the study of (2); substituting $\tan \theta_{k B}=k_{\perp} / k_{\|}$, it can be rewritten

$$
\tan ^{2} \theta_{k B}=\frac{1}{2} \frac{A_{p}-1}{A_{p}^{2}\left(1-\frac{\gamma}{k_{\|} V_{t h \|}}\left(\frac{\pi}{2}\right)^{\frac{1}{2}}\right)-A_{p}-1} .
$$

The propagation angle is therefore an explicit function of $A_{p}$ parameterized by the normalized growth rate $\gamma / k_{\|} V_{t h \|}$. In the case of realistic conditions (not too large an anisotropy), the term containing $\gamma$ can be neglected with respect to 1 . Consequently, it appears that $\theta_{k B}$ (like $\tan ^{2} \theta_{k B}$ ) is a decreasing function of the anisotropy. For larger anisotropies, the term containing $\gamma$ is no longer negligible; thus the $A_{p}^{2}$ contribution in the denominator decreases which slows down the $\theta_{k B}$ decrease. In that case, however, the relation 
is no longer explicit as $\theta_{k B}$ intervenes in the $\gamma$-related term $\left(k_{\|}=k \cos \theta_{k B}\right)$. A restriction to the use of (4) is also that it is only valid for $k \rho_{i} \ll 1$ (which is not the case for $A_{p}>3$ at maximum growth). The analysis still stands for larger anisotropy, but no simple analytic expression can be obtained. The results presented in the left-hand panels of Figure 2 can be synthesized as follows: for increasing values of the proton anisotropy $k, \gamma$ and $\left|\delta B_{y}\right| /|\delta \vec{B}|$ increase, whereas $\theta_{k B}$ decreases.

The growth of the mirror mode is linked not only to the anisotropy but also to the plasma $\beta$, the ratio between the kinetic pressure and magnetic pressure. As discussed in the Introduction, the mirror mode develops in environment where the anisotropy is low $\left(A_{p} \simeq 2\right)$ and the plasma $\beta$ is high. We thus show on the right of Figure 2 the role of $\beta_{\| p}$ on the same quantities as previously. Ion and electron temperatures as well as anisotropies are kept constant (we choose $A_{p}=2, T_{e} / T_{\| p}=0.01$ ), while the value of the ambient magnetic field progressively decreases. It thus allows $\beta_{\| p}$ to vary, from 1 to 25 , in our analysis. The results are displayed in Figures 2d, 2e, and $2 \mathrm{f}$.

The close similarities in the variations of the different quantities $\left(P, k, \gamma, \theta_{k B}\right.$, and $\left.\left|\delta B_{y}\right| /|\delta \vec{B}|\right)$ with either the anisotropy or the plasma $\beta$ may be a consequence of changes in the Larmor radius, which increases with the anisotropy and the plasma $\beta$ (for constant $T_{\| p}$ which was the choice in the two studies conducted here). For this second study the results are qualitatively similar to the first one and can be synthesized as follows: for increasing values of the $\beta_{\| p} k, \gamma$ and $\left|\delta B_{y}\right| /|\delta \vec{B}|$ increase, whereas $\theta_{k B}$ decreases.

\subsection{Effects of the Electron Anisotropy}

The electron anisotropy is generally less than that of ions (in typical regions of interest like the magnetosheath) and consequently its role in the mirror mode growth is less important. However, as the mirror mode growth rate $\gamma$ increases with the number of anisotropic species, the combining effect of both anisotropies may enhance the value of the Alfvénic component. Therefore we briefly address here the question of the effect of electron anisotropy alone on the Alfvénic component of the magnetic perturbation and on the growth rate. These quantities are plotted in Figures $3 \mathrm{a}$ and $3 \mathrm{~b}$, respectively, for electron anisotropies varying from 1.8 to $6, A_{p}=2$ and for two cases: $T_{\| e} / T_{\| p}=0.01$ and 0.1 (which is a more typical value for the magnetosheath). In the first case, the curve in Figure 3a presents a smooth increasing trend, but the ratio of the Alvénic component to the magnetic amplitude does not exceed $4 \%$, whereas it in- 
creases by $\sim 2$ orders of magnitude and reaches $30 \%$ for the same range of varying ion anisotropies (see Figure 2c). In a similar way the growth rate increases slightly but still remains a small fraction (less than $2 \%$ ) of the ion cyclotron frequency. In the second case the slopes of the two curves are largely enhanced denoting a much more important influence of $A_{e}$ than in the previous case. The growth rate is doubled between $A_{e}=1.8$ and $A_{e}=3$, whereas $\left|\delta B_{y}\right| /|\delta \vec{B}|$ is increased by more than $50 \%$ for the same range of anisotropies. However, the Alfvénic component still remains less than $6 \%$ of the total amplitude. In both cases the propagation angle and the wave vector at maximum growth are not sensitive functions of the anisotropy. For larger anisotropy $\left(A_{e}>3\right)$ the behavior of the mirror mode is perturbed: the dispersion curve $(\omega, k)$ is no longer bell-like shaped with a single maximum because a new mode begins to grow at $k \rho_{e} \simeq 1$. This mode requires more investigations which may hardly be tractable analytically as expansion in the small parameter $k \rho_{i}$ are not possible. However, for magnetosheath studies, $A_{e}=3$ is still a large value, and we can restrain our analysis to smaller anisotropies.

In conclusion, we can affirm that the electron temperature effect is important on the variations with the electron anisotropy of the magnetic Alfvénic component and of the growth rate values, although these values remain negligible in comparison with the ones obtained in the previous section, at least in the magnetosheath where the anisotropy is not too large. Therefore the electron anisotropy is not a main parameter in the generation of the Alfvénic component of the magnetic perturbation. The influence of finite $T_{e}\left(T_{\| e} / T_{\| p}\right.$ varying over several orders of magnitude) on the mirror instability growth rate is investigated in more detail by Pokhotelov et al. [2000].

\subsection{Effects on the Velocities and the Deviation From MHD}

A MHD treatment does not allow the existence of a torsional component for the magnetic field. This is contradicted both by the kinetic analysis of the previous section and by the data analysis of the next section. However, under typical magnetosheath conditions, the torsional magnetic field component is generally small. In the same way, MHD gives a zero Alfvénic component of the velocity. By contrast, the kinetic results in Figure 4 show that this component is indeed the largest. We shall see later that, as given by Lin et al. [1998], observations support these results.

Regarding the variations of the ratio of the ion Alfvénic 
velocity perturbation to the total amplitude (Figure 4 , top), there is a decreasing trend with increasing anisotropies. However, the values represent at least $89 \%$ (in the case $A_{e}=1, A_{p}=6$ ) which is a clear indication that the Alfvénic component is always prevalent. There is a similar behavior in the case of varying electron anisotropy, but the decrease is only a few percent (Figure 4, bottom). The ratio of the Alfvénic component to the total amplitude remains above $94 \%$ for the electron anisotropies chosen. It should be noted that the total amplitude also decreases with the anisotropy, by a factor $\sim 10$ between $A_{p}=1.8$ and $A_{p}=6$. The behavior of the Alfvénic component of the electron velocity is reversed and this is shown with dash curves. The component increases with both anisotropies, the variations being greater with the electron anisotropy. However, in this case the dominant component is no longer the Alfvénic one but the $z$ one (not shown), parallel to the ambient magnetic field. The increase of the Alfvénic component does not modify this predominance, although the ratio of Alfvénic component over the total velocity amplitude reaches 0.25 for $A_{p}=6$ and 0.38 for $A_{e}=6$.

In short, it appears that the ion torsional velocity component is not significantly affected by the variations of anisotropies, unlike the electron component which can be enhanced by 1 order of magnitude. However, within the range of magnetosheath-like values of the anisotropy variations $\left(A_{p, e} \leq 2\right)$, it is possible to affirm that the velocity properties of the kinetic mirror mode do not change much. An interesting finding of this study is the predominance of the Alfvénic velocity component of the ions over the other components $\left(\delta V_{p y} / \delta V_{p} \simeq 0.9\right)$. This clearly shows the limitation of the MHD analysis in which this component is always zero.

This important difference between MHD and kinetic theories is not yet fully understood. An explanation can be proposed following the work of Southwood and Kivelson [1993, equation 19]. They show that the perturbation of the distribution function is composed of two terms, the second of which being an additional contribution from the kinetic treatment. This term indeed contains the effect of the resonant particles (with $\left.v_{\|}=0\right)$ which are the cause of the difference between the two theories in the dispersion relation, and in derived perturbed moments.

Although the work of Lin et al. [1998] relies on $\delta V_{p y} \neq 0$, without theoretical explanation, we have verified its main ingredient: the $90^{\circ}$ phase difference between the magnetic field and, specifically, the Alfvénic 
velocity fluctuations for the mirror mode. Indeed, in our analysis, the phase of the (ion and electron) velocity components were found to be either $0^{\circ}$ or $180^{\circ}$, whereas the component of the magnetic field perturbations are $\pm 90^{\circ}$ (the normalization in the study is such that the phase of $\delta E_{x}$ is 0$)$. These phase relations also imply that the ratio $i \delta V_{\xi} / \delta V_{y}$ is purely imaginary denoting a linear polarization, similarly to what has been found for magnetic perturbations. The mass conservation which, for the small-amplitude mirror mode perturbations, can be written

$$
\frac{\delta n}{n_{0}}=-i \frac{\delta V_{k}}{\gamma / k},
$$

is verified in our analysis. Thus the density oscillations are related to the velocity perturbations along the wave vector; and these quantities also show a $90^{\circ}$ phase difference.

\section{Experimental Tests}

\subsection{Mirror Events}

We shall use different well-identified mirror mode events in order to show whether or not the kinetic properties of the mirror mode previously discussed are seen in observations. The identification has been done by several authors, using the $B-n$ anticorrelation and transport ratios to distinguish mirror from slow modes.

In order to achieve the principal goal of this section (revealing Alfvénic component of the perturbations), we first need to transform the data into a suitable frame, that is, the one presented in section 2. The transformation requires the knowledge of the direction of the wave vector $\vec{k}$. This first step is not an easy one as the most common method to determine $\vec{k}$, the minimum variance analysis (MVA [Sonnerup and Cahill, 1967]), fails in the case of linearly polarized mode. Indeed, for such mode the MVA leads to uncertainty as it is impossible to distinguish between the minimum and intermediate directions (they degenerate); only the maximum variance direction is well defined. The former remark is exploited in an alternative method using two spacecraft and developed by Chisham et al. [1999] which we shall use and discuss in the following. As the MVA is very sensitive to the low-frequency inhomogeneities of the field, we filtered the data by removing all fluctuations of period $120 \mathrm{~s}$ and above (the period of the mirror mode fluctuations are $\sim 30 \mathrm{~s}$ ). We used the highest time resolution when available $(0.0315 \mathrm{~s}$, or else $5 \mathrm{~s})$.

We derive a criterion in order to establish whether a mode is linearly or elliptically polarized. This is done 
based on MVA eigenvalues ratios. A linearly polarized structure is characterized by a well-defined maximum variance direction (i.e., $\lambda_{\text {int }} \ll \lambda_{\max }$ ), whereas the two other directions are less clearly separated (i.e., $\left.\lambda_{\min } \leq \lambda_{\text {int }}\right)$. The requirement for a mode to be linearly polarized can be formalized as follows:

$$
\lambda_{\text {int }} / \lambda_{\max } \leq 0.2 \text { and } \lambda_{\min } / \lambda_{\text {int }} \geq 0.3,
$$

where the values 0.2 and 0.3 have been found by trial and error. When eigenvalues do not meet the above requirements, we classify the wave "elliptic".

A few mirror mode events have been gathered in Table 1 which also displays some of the mode characteristics: $A_{p}, \beta_{\| p}, \lambda_{\text {int }} / \lambda_{\max }, \lambda_{\min } / \lambda_{\text {int }}$, polarization, and waveform. They come from the time period (September 1, 1984, November 30,1984) of the AMPTE data set and have been recorded by United Kingdom Satellite (UKS) and/or Ion Release Module (IRM). The eigenvalues $\lambda$, calculated by MVA, have been used to discriminate the wave polarization in agreement with criterion 6 . The plasma parameters $A_{p}$ and $\beta_{\| p}$ have been calculated from the particle and magnetic experiments on board the spacecraft. The waveform classification refers to the shape of the main component of the magnetic field perturbation $\left(\delta B_{\xi}\right)$. It is a more subjective characteristic, as no proper criterion defines it. However, it was possible to distinguish two other categories, added to the classical picture of sinusoidal oscillations ("S"), termed "U" and "D" for spiky up and down, respectively. An example of spiky down signature is given on the top panel of Figure 5 where one can see largeamplitude negative peaks superimposed on a smoother background (the negative peaks are larger in magnitude than the positive ones); as time evolved, on this particular event, the waveform tends to be more sinusoidal. A spiky up event is the reversed situation. We shortly discuss below the interest of these characteristics.

\subsection{First Example: A Linearly Polarized Event}

We chose an event from September 21, 1984 (case 2). We use Chisham et al.'s [1999] method on the whole interval (1313-1336 UT) to find $\vec{k}$. The angle between $\vec{k}$ and $\vec{e}_{\min }$ (minimum variance direction) is $42.4^{\circ}$. We can then express the magnetic field and velocity perturbations in the coordinate system $(\hat{\xi}, \hat{y}, \hat{k})$. These are shown in Figures 5 and 6 . For comparison, Figure 5 also shows the $k$ component of the magnetic perturbations computed from a MVA wave vector determination (last panel). 
Two remarks can be made. First, $\delta B_{y}$ is the same order of magnitude as $\delta B_{k}$ with the two-satellite method, but it is a bit larger with MVA; second, $\delta V_{y}$ is not the largest component with the two-satellite method $\left(\left(<\delta V_{i y}^{2}>/<\delta V_{i}^{2}>\right)=0.49\right)$, but it is with MVA

$\left(\left(<\delta V_{i y}^{2}>/<\delta V_{i}^{2}>\right)=0.58\right)$. This shows the difficulty of the choice of an adequate method for the wave vector determination in the case of a linearly polarized event. The limitations of the MVA have been discussed earlier; in the case of Chisham et al.'s [1999] method, limitations may be found in the fact that it requires some external parameters (separation vector, magnetosheath flow velocity, time lag between the two satellites) which can also introduce significant errors. However, two parameters should not lead to errors: first, the maximum variance direction is well defined, and second, it can be shown that, in the case of the mirror mode $\left(\omega_{\text {rest }}=0\right)$, the value of the observed frequency $\omega_{s c}$, even if it is difficult to determine, does not influence the direction of $\vec{k}$ (it only modifies the magnitude $k)$.

For the present event, in the case of the magnetic field, it is impossible to affirm whether or not the signal is actually the Alfvénic component or noise inherent in the method. The $\beta_{\| p}$ is indeed less than 1 and, although the anisotropy is $\sim 2$, from our previous analysis this should not lead to $\delta B_{y} / \delta B$ greater than 0.02 . $\mathrm{Up}$ to now, our research of a clear case $\left(\delta B_{y}\right.$ clearly distinct from the noise) has been unfruitful, partly because of the difficulty to find a good conjunction for which it was possible to extract the relevant parameters from the high-resolution data sets (when available) of both satellites, and partly because our data set does not present cases with sufficiently high values of the two key parameters, $\beta_{\| p}$ and $A_{p}$. As far as velocity perturbations are concerned, the calculated ratio $\left(<\delta V_{i y}^{2}>/<\delta V_{i}^{2}>\right)^{1 / 2}$ is generally less than the value shown in the analysis $(\sim 0.9)$.

Although we have shown that the mirror mode should be theoretically linearly polarized, the observations do not follow this strict rule. As we said earlier in this section, some events present a departure from a linear polarization, and, as far as the waveform is concerned, some events are far from the classical view of a sinusoidal magnetic signature. These two points are addressed in the next section.

\subsection{Second Example: An Elliptically Polarized Event}

From Table 1, one sees that elliptically polarized events (as defined by the criterion 6) occur more fre- 
quently than linearly polarized ones. We choose case 10 as an example. We use MVA to determine $\vec{k}$ and rotate the magnetic field and velocity perturbations in the coordinate system shown in Figure 1 . The corresponding time series are plotted in Figure 7 . It is not surprising that such a case presents a nonvanishing $\delta B_{y}$, even larger that the one predicted from the kinetic theory with the corresponding plasma parameters $\left(\delta B_{y} / \delta B \leq 0.1\right)$; an elliptically polarized wave must indeed present two main components. Concerning the velocity components, we see that $\delta V_{i \xi}$ and $\delta V_{i y}$ are of the same order of magnitude.

What are the origins of these elliptical modes which depart from the kinetic linear theory? First, it is true that the magnetosheath plasma is highly inhomogeneous, and a more complete theoretical treatment may considerably modify our conclusions based on homogeneous plasma. Other studies [Chisham et al., 1999; Balikhin et al., 2001] suggest that, at least sometimes, modes which appear in most other respects to be mirror modes have a finite rest frame frequency (and phase velocity). This has been already pointed out theoretically by Hasegawa [1969], and more recently by Johnson and Cheng [1997]. These authors show that the existence of gradients of the magnetic field and/or the density leads, for the mirror mode, to a finite frequency, a combination of the diamagnetic drift frequency and the Doppler shift frequency associated with plasma flow. They claim that large gradients near the magnetosheath boundaries globally determine the structure of the mirror modes observed inside the magnetosheath. Second, observations often show large perturbation amplitudes $(\delta B / B \simeq 0.5)$ which may lead to nonlinear effects not described here as well. Third, the superposition of two strictly linearly polarized mirror modes may result in a signature that the MVA cannot separate from an elliptically polarized mode; and in this case the observed frequency could remain zero. This shows that the conclusion we drew in the beginning of section 3 (the ellipticity of a wave mode implies $\omega \neq 0$ ) is only valid for a single mode. These remarks emphasize the need for multipoints measurements which will enable studies of the 3-D structure of the mirror mode. The few existing works which depart from the planar wave model indeed suggest that such studies may provide answers to present discrepancies between models and observations.

The waveforms are also interesting: we observed that, far from being uniformly sinusoidal, mirror modes present a variety of waveforms, for which the anticorrelation between the magnetic field and plasma perturbations persists. The relation of these waveforms 
with other parameters (position, time, plasma,...) is currently under investigation; no link has been yet established between the wave form and the observation position relative to the magnetopause and bow shock. These waveforms may be related to the saturation of the mirror mode as discussed by Kivelson and Southwood [1996] (see also Winterhalter et al. [1994]).

\section{Conclusion}

In this article we emphasize the limits of the MHD treatment of the mirror mode. In particular, we have shown that the kinetic mirror mode possesses torsional components of both magnetic and velocity fields which are forbidden in MHD treatments. The parameter analysis revealed that the values of these components are sensitive functions of both the anisotropy and the plasma to magnetic pressure ratio. In magnetosheath plasma where $A_{p} \simeq 2$, the torsional component of the magnetic field can reach a third of the field amplitude for high $\beta\left(\beta_{\| p} \simeq 10\right)$ but is marginal for values less than 3. By contrast the torsional component of the ion velocity is in fact always the largest one. Still in the magnetosheath context, we have shown that the influence of the electron anisotropy is of minor importance for these conclusions; we noted that the electron to ion temperature ratio can significantly modify the growth rate and the torsional magnetic component variations. Finally, we have also verified the $90^{\circ}$ phase difference between magnetic and plasma velocity fluctuations anticipated by Lin et al.'s [1998] analysis for both electrons and ions.

An important finding of the study is that the mirror mode should remain linearly polarized. Thus for increasing anisotropy the progressive appearance of the torsional component does not lead to a rotating magnetic field but to a tilt out of the coplanarity plane. The implication of this polarization is that minimum variance analysis (and coplanarity theorem) must not be used to determine the wave vector direction since the minimum and intermediate variance direction cannot be accurately separated. Unfortunately, the determination of $\vec{k}$ is important to define the coplanarity plane, and consequently, the Alfvénic direction. An alternative method is presented by Chisham et al. [1999] using two satellites.

However, our data analysis of several identified mirror mode events raised many questions. We found that several cases are distinctly not linearly polarized. For instance, following our classification criterion, the event chosen by Lin et al. [1998] falls in this category. In such a case, it is then not striking to find a nonvanishing tor- 
sional component which is larger than the one of a linearly polarized mode with equivalent plasma conditions $\left(\beta_{\| p}\right.$ and $\left.A_{p}\right)$. Observed linearly polarized cases have also been found, but the plasma conditions were such that the values of the predicted and observed torsional components were not distinguishable from the noise.

Progress in understanding the behavior of the mirror mode in the magnetosheath, and in particular its 3-D structure, requires multipoints measurements (to determine $\vec{k}$ and $\omega$ to high accuracy) for clear examples of both linear and elliptic polarization and for the various waveforms described here. Such data, soon available thanks to the Cluster mission, will provide a reliable test bed against which future theoretical works, including inhomogeneous media and large-mode amplitudes studies, can be developed and tested.

Acknowledgments. V.G. is supported by a UK PPARC grant. Thanks to AMPTE PI's and ISSI (Bern, Switzerland).

Janet G. Luhmann thanks Gérard Belmont and another referee for their assistance in evaluating this paper.

\section{References}

Balikhin, M. A., S. Schwartz, S. N. Walker, H. S. C. K. Alleyne, and H. Lühr, Dual spacecraft observations of standing waves in the magnetosheath, J. Geophys. Res., in press, 2001.

Barnes, A., Collisionless heating of the solar-wind plasma, 1 , Theory of the heating of collisionless plasma by hydromagnetic waves, Astrophys. J., 154, 751, 1968.

Belmont, G., and C. Mazelle, Polytropic indices in collisionless plasmas: Theory and measurements, J. Geophys. Res., 97, 8327, 1992.

Belmont, G., D. Hubert, C. Lacombe, and F. Pantellini, Mirror mode and other compressive ULF modes, in Proceedings of the 26th ESLAB Symposium., Eur. Space Agency Spec. Publ., ESA SP-346, 1992.

Chisham, G., S. J. Schwartz, M. A. Balikhin, and M. W. Dunlop, AMPTE observations of mirror mode waves in the magnetosheath: Wave vector determination, J. Geophys. Res., 104, 437, 1999.

Denton, R. E., S. P. Gary, X. Li, B. J. Anderson, J. W. Labelle, and M. Lessard, Low-frequency fluctuations in the magnetosheath near the magnetopause, J. Geophys. Res., 100, 5665, 1995.

Fazakerkey, A. N., and D. J. Southwood, Theory and observations of magnetosheath waves, in Solar Wind Sources of Magnetospheric Ultra-Low-Frequency Waves, Geophys. Monogr. Ser., vol. 81, edited by M. J. Engebretson, p. 147, AGU, Washington, D. C., 1994.

Fried, B. D., and S. D. Conte, The Plasma Dispersion Function, Academic, San Diego, Calif., 1961.

Gary, S. P., The mirror and ion cyclotron anisotropy instabilities, J. Geophys. Res., 97, 8519, 1992.

Glassmeier, K.-H., U. Motschmann, C. Mazelle, F. Neubauer, K. Sauer, S. Fuselier, and M. Acuna, Mirror 
modes and fast magnetoacoustic waves near the magnetic pileup boundary of comet P/Halley, J. Geophys. Res., 98, 20,955, 1993.

Hasegawa, A., Drift mirror instability in the magnetosphere, Phys. Fluids, 12, 2642, 1969.

Hasegawa, A., Plasma Instabilities and Nonlinear Effects, Springer-Verlag, New York, 1975.

Hubert, D., C. Lacombe, C. C. Harvey, M. Moncuquet, C. T. Russel, and M. F. Thomsen, Nature, properties, and origin of low-frequency waves from an oblique shock to the inner magnetosheath, J. Geophys. Res., 103, 26,783, 1998.

Huddleston, D. E., R. J. Strangeway, X. Blanco-Cano, C. T. Russell, M. G. Kivelson, and K. K. Khurana, Mirror-mode structures at the Galileo-Io flyby: Instability criterion and dispersion analysis, J. Geophys. Res., 104, 17,479, 1999.

Johnson, J. R., and C. Z. Cheng, Global structure of mirror modes in the magnetosheath, J. Geophys. Res., 102, 7179, 1997.

Kivelson, M. G., and D. J. Southwood, Mirror instability; 2, The mechanism of nonlinear saturation, J. Geophys. Res., 101, 17,365, 1996.

Krauss-Varban, D., N. Omidi, and K. B. Quest, Mode properties of low-frequency waves: Kinetic theory versus HallMHD, J. Geophys. Res., 99, 5987, 1994.

Lacombe, C., and G. Belmont, Waves in the Earth's magnetosheath: Observations and interpretations, Adv. Space Res., 15(8/9), 329, 1995.

Lacombe, C., F. G. E. Pantellini, D. Hubert, C. C. Harvey, A. Mangeney, G. Belmont, and C. T. Russel, Mirror and Alfvénic waves observed by ISEE 1-2 during crossings of the Earth's bow shock, Ann. Geophys., 10, 772, 1992.

Lin, C.-H., J. K. Chao, L. C. Lee, D. J. Wu, Y. Li, B. H. Wu, and P. Song, Identification of mirror waves by the phase difference between perturbed magnetic field and plasmas, J. Geophys. Res., 103, 6621, 1998.

McKean, M. E., D. Winske, and S. P. Gary, Kinetic properties of mirror waves in magnetosheath plasmas, Geophys. Res. Lett., 19, 1331, 1992.

Pantellini, F. G. E., and S. J. Schwartz, Electron temperature effects in the linear proton mirror instability, J. Geophys. Res., 100, 3539, 1995.

Pokhotelov, O. A., M. A. Balikhin, H. Alleyne, and O. Onishchenko, Mirror instability with finite electron temperature effects, J. Geophys. Res., 105, 2393, 2000.

Rönnmark, K., WHAMP: Waves in Homogeneous, Anisotropic Multicomponent Plasmas, Rep. 179, Kiruna Geophys. Inst., Kiruna, Sweden, 1982.

Russell, C. T., W. Riedler, K. Schwingenschuh, and Y. Yeroshenko, Mirror instability in the magnetosphere of comet Halley, Geophys. Res. Lett., 14, 644, 1987.

Song, P., C. T. Russel, and S. P. Gary, Identification of lowfrequency fluctuations in the terrestrial magnetosheath, J. Geophys. Res., 99, 6011, 1994.

Sonnerup, B. U. O., and L. J. Cahill Jr., Magnetopause structure and attitude from Explorer 12 observations, $J$. Geophys. Res., 72, 171, 1967.

Southwood, D. J., and M. G. Kivelson, Mirror instability; 1, Physical mechanism of linear instability, J. Geophys. Res., 98, 9181, 1993.

Winterhalter, D., M. Neugebauer, B. Goldstein, E. Smith, S. Bame, and A. Balogh, Ulysses field and plasma ob- 
servations of magnetic holes in the solar wind and their relation to mirror-mode structures, J. Geophys. Res., 99, 23,371, 1994.

M. Balikhin, Department of Automatic Control and Systems Engineering, Sheffield University, P.O. Box 600, Mappin Street, Sheffield S1 4DU, England, UK. (m.balikhin@sheffield.ac.uk)

T. M. Bauer, Max-Planck-Institut für extraterrestrische Physik, Postfach 1603, D-85740 Garching, Germany. (thb@mpe.mpg.de)

M. Dunlop, Space and Atmospheric Physics, Imperial College, South Kensington, London SW7 2AZ, England, UK. (m.dunlop@ic.ac.uk)

V. Génot and S. J. Schwartz, Astronomy Unit, Queen Mary, University of London, Mile End Road, London E1 4NS, England, UK. (v.genot@qmw.ac.uk; s.schwartz@qmw.ac.uk)

C. Mazelle, Centre d'Étude Spatiale des Rayonnements, CNRS, 9 Av. Colonel Roche, 31400 Toulouse, France. (mazelle@cesr.fr)

(Received December 13, 2000; revised May 2, 2001; accepted May 2, 2001.)

\footnotetext{
${ }^{1}$ Astronomy Unit, Queen Mary, University of London, London, England, United Kingdom

${ }^{2}$ Centre d'Etude Spatiale des Rayonnements, Toulouse, France

${ }^{3}$ Department of Automatic Control and Systems Engineering, Sheffield University, Sheffield, England, United Kingdom

${ }^{4}$ Space and Atmospheric Physics, Imperial College, London, England, United Kingdom

${ }^{5}$ Max-Planck-Institut für Extraterrestrische Physik, Garching, Germany
}

Copyright 2001 by the American Geophysical Union.

Paper number 2000JA000457.

0148-0227/01/2000JA000457\$09.00 


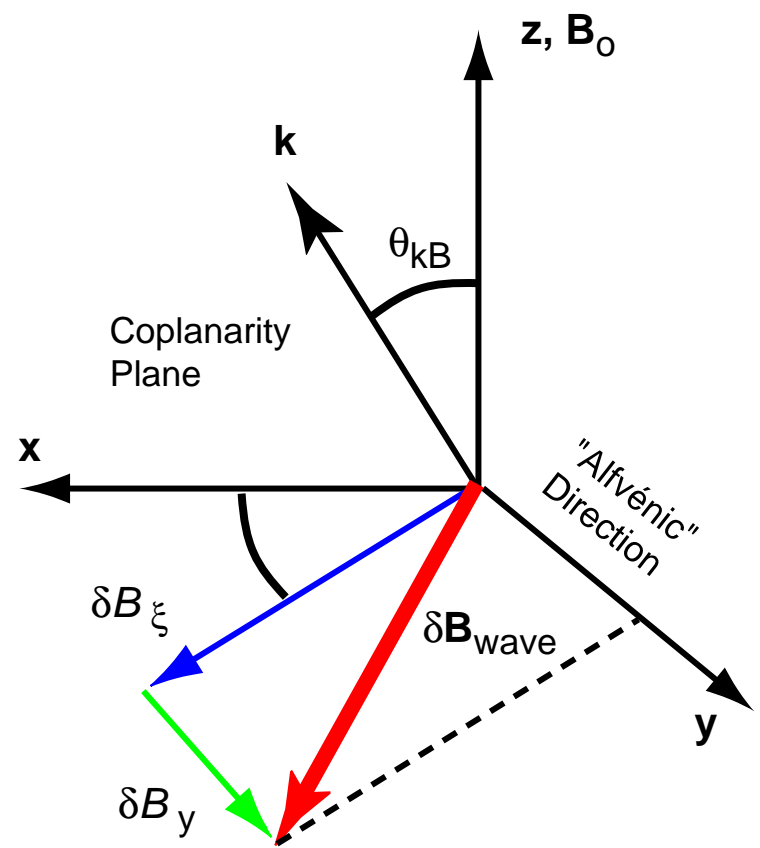

Figure 1. Sketch of the coordinate system used throughout the study. The Alfvénic direction is chosen to be $\hat{y}$ and is perpendicular to the coplanarity plane which contains $\vec{B}_{0}$ and $\vec{k}$.

Figure 1. Sketch of the coordinate system used throughout the study. The Alfvénic direction is chosen to be $\hat{y}$ and is perpendicular to the coplanarity plane which contains $\vec{B}_{0}$ and $\vec{k}$. 
Figure 2. Variations at maximum growth rate with the (left) proton anisotropy and with (right) parallel proton $\beta$, of different key quantities: (top) the polarization, (middle) the wavenumber, normalized to the inverse of the proton Larmor radius, and growth rate, normalized to the proton gyrofrequency, and (bottom) the propagation angle and the ratio of the torsional component value to the total magnetic amplitude.

Figure 2. Variations at maximum growth rate with the (left) proton anisotropy and with (right) parallel proton $\beta$, of different key quantities: (top) the polarization, (middle) the wavenumber, normalized to the inverse of the proton Larmor radius, and growth rate, normalized to the proton gyrofrequency, and (bottom) the propagation angle and the ratio of the torsional component value to the total magnetic amplitude.
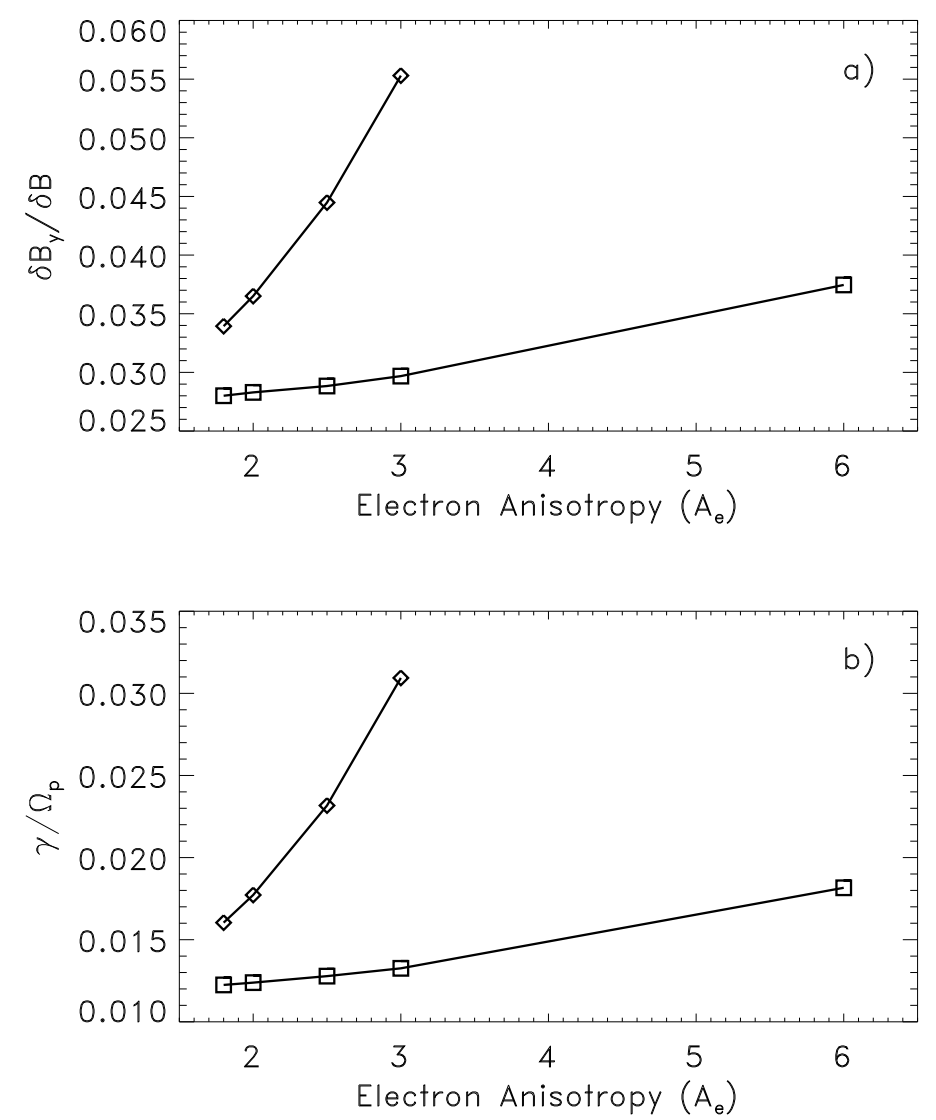

Figure 3. Variations with the electron anisotropy for $A_{p}=2$ of (a) the ratio of the Alfvénic component to the amplitude of the magnetic field perturbation and (b) the growth rate of the mirror mode. The curves are plotted for different temperature ratios: $T_{\| e} / T_{\| p}=0.01$ (square) and $T_{\| e} / T_{\| p}=0.1$ (diamond).

Figure 3. Variations with the electron anisotropy for $A_{p}=2$ of (a) the ratio of the Alfvénic component to the amplitude of the magnetic field perturbation and (b) the growth rate of the mirror mode. The curves are plotted for different temperature ratios: $T_{\| e} / T_{\| p}=0.01$ (square) and $T_{\| e} / T_{\| p}=0.1$ (diamond). 

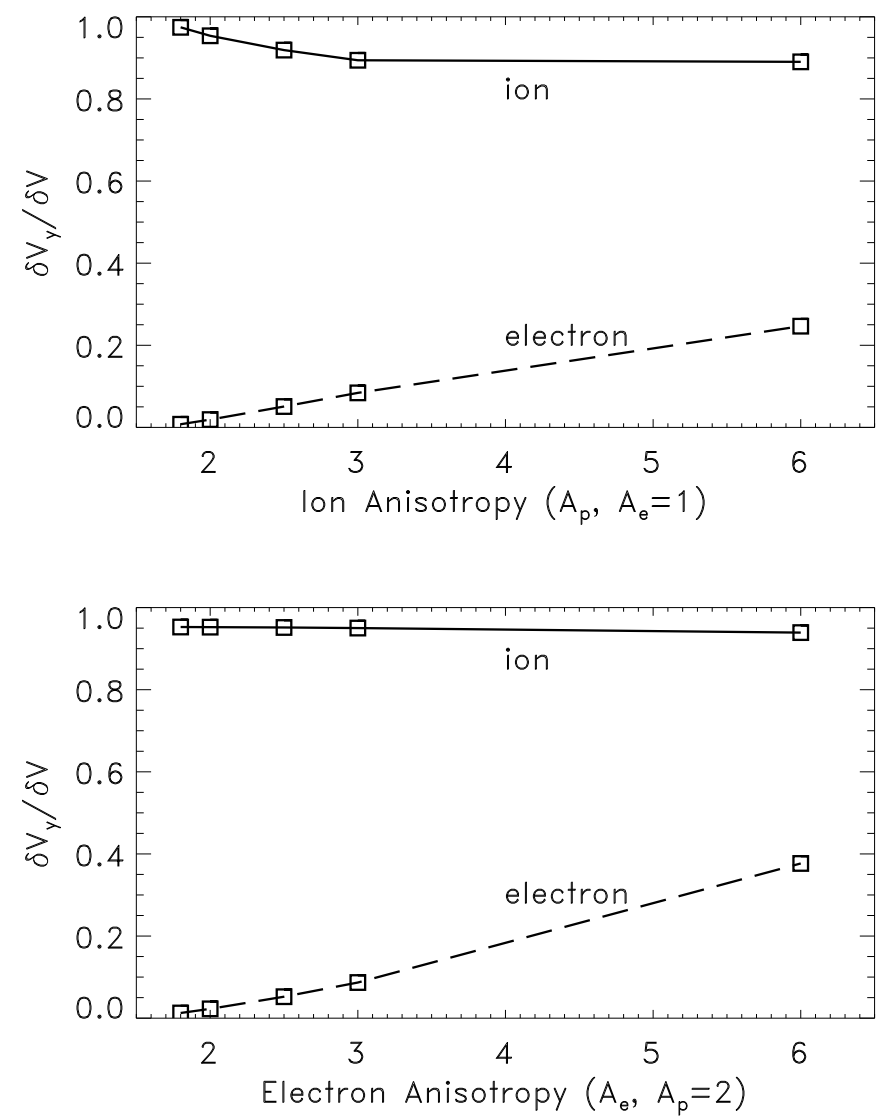

Figure 4. Alfvénic component of the ion (solid lines) and electron (dashed lines) velocities as a function of the (top) ion and (bottom) electron anisotropies. Values are normalized to the total amplitude for each species $\left(\beta_{\| p}=1, T_{\| e} / T_{\| p}=0.01\right)$.

Figure 4. Alfvénic component of the ion (solid lines) and electron (dashed lines) velocities as a function of the (top) ion and (bottom) electron anisotropies. Values are normalized to the total amplitude for each species $\left(\beta_{\| p}=1, T_{\| e} / T_{\| p}=0.01\right)$. 


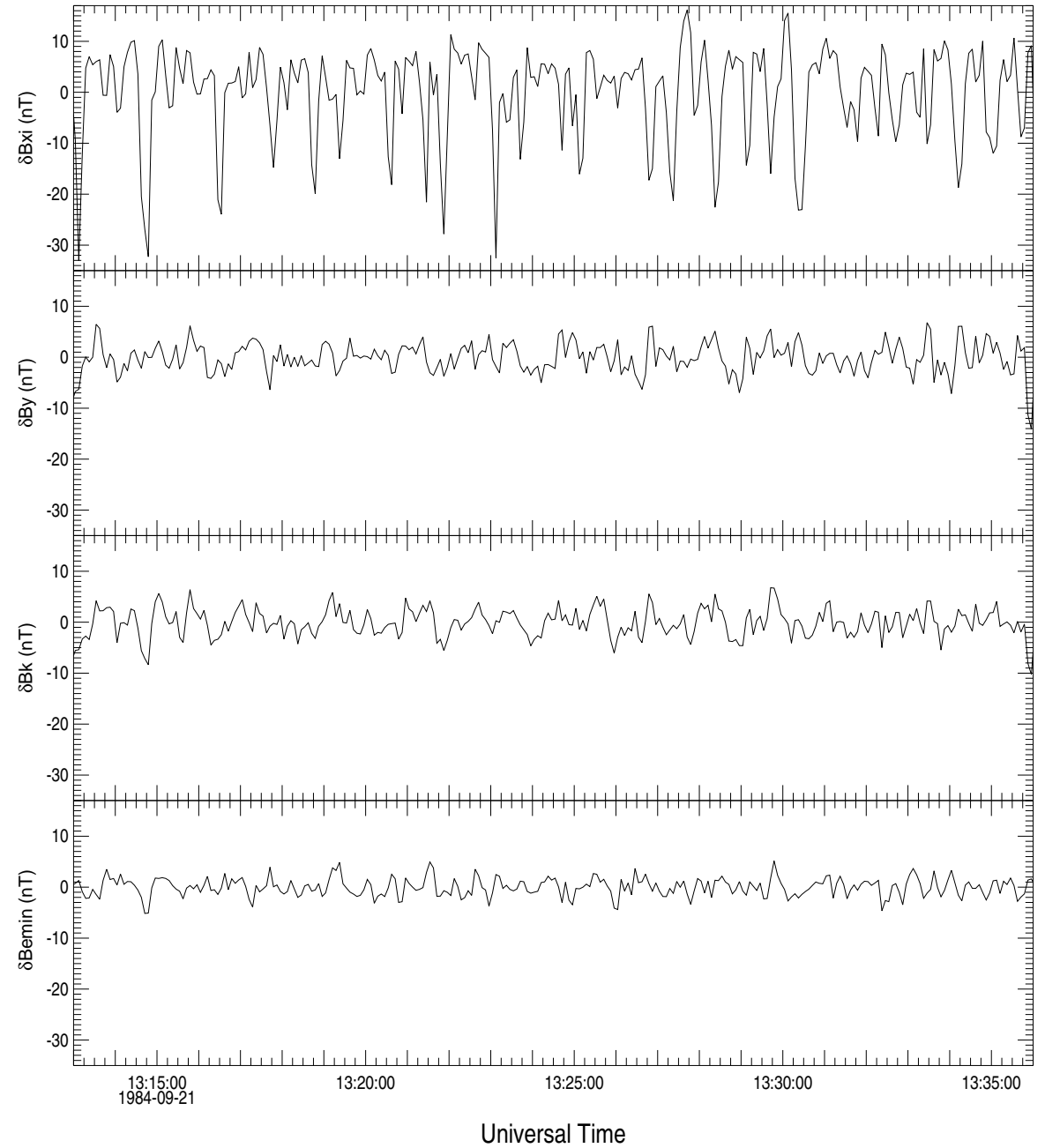

Figure 5. Magnetic field perturbations of the September 21, 1984 mirror event rotated to the coordinate system $(\hat{\xi}, \hat{y}, \hat{k})$ presented in Figure 1 . Variable $\hat{k}$ is determined by the two-satellite method. The last panel is the perturbation in the minimum variance direction. 
Figure 5. Magnetic field perturbations of the September 21, 1984 mirror event rotated to the coordinate system $(\hat{\xi}, \hat{y}, \hat{k})$ presented in Figure 1 . Variable $\hat{k}$ is determined by the two-satellite method. The last panel is the perturbation in the minimum variance direction.

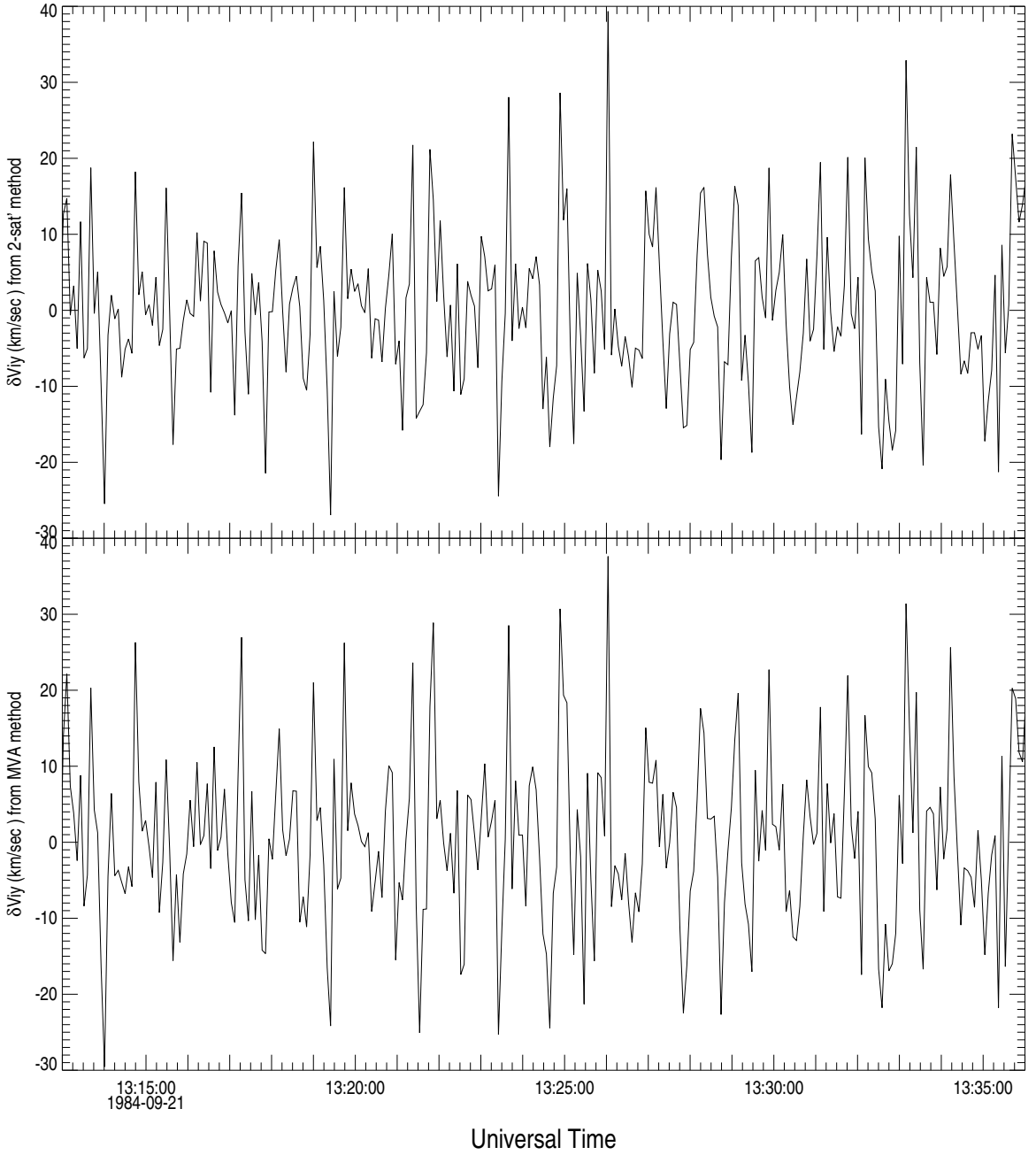


Figure 6. Ion velocity perturbations of the September 21, 1984 mirror event rotated to the coordinate system $(\hat{\xi}, \hat{y}, \hat{k})$ presented in Figure 1 . Variable $\hat{k}$ is determined by the (top) two-satellite method and by (bottom) MVA.

Figure 6. Ion velocity perturbations of the September 21, 1984 mirror event rotated to the coordinate system $(\hat{\xi}, \hat{y}, \hat{k})$ presented in Figure 1. Variable $\hat{k}$ is determined by the (top) twosatellite method and by (bottom) MVA.

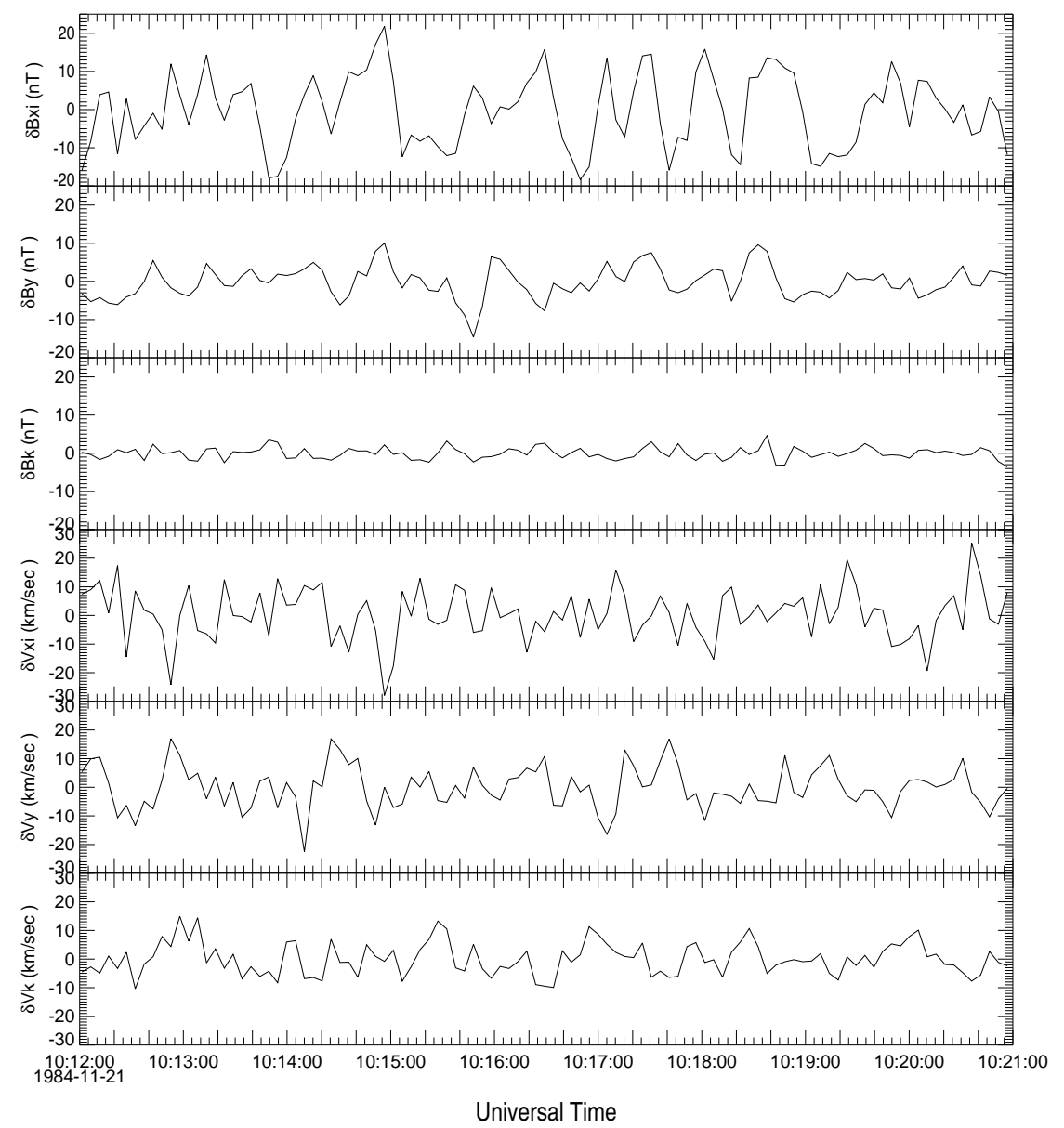


Figure 7. Magnetic field perturbations (top three panels) and ion velocity (bottom three panels) of the November 21, 1984 mirror event rotated to the coordinate system $(\hat{\xi}, \hat{y}, \hat{k})$ presented in Figure 1. Variable $\hat{k}$ is determined by the MVA.

Figure 7. Magnetic field perturbations (top three panels) and ion velocity (bottom three panels) of the November 21, 1984 mirror event rotated to the coordinate system $(\hat{\xi}, \hat{y}, \hat{k})$ presented in Figure 1. Variable $\hat{k}$ is determined by the MVA.

Table 1. Mirror Mode Events From AMPTE. Abbreviations are n.a., not available; E/L, elliptically/linearly polarized; S, sinusoidal; U/D, spiky up/down; 1, IRM; 2, UKS. Case 8 presents a large gap in data.

\begin{tabular}{rcccccccccc}
\hline Case & Day in 1984 & Start, UT & Stop, UT & $A_{p}$ & $\beta_{\| p}$ & $\lambda_{\text {int }} / \lambda_{\max }$ & $\lambda_{\min } / \lambda_{\text {int }}$ & Polarized & Waveform & Spacecraft \\
\hline 1 & Sept. 1 & $0642: 00$ & $0718: 00$ & 1.5 & 1.1 & 0.27 & 0.21 & $\mathrm{E}$ & $\mathrm{S}$ & 1 and 2 \\
2 & Sept. 21 & $1313: 00$ & $1336: 00$ & 2 & 0.4 & 0.18 & 0.28 & $\mathrm{~L}$ & $\mathrm{D}$ & 1 and 2 \\
3 & Sept. 21 & $1426: 00$ & $1444: 00$ & 1.4 & 1.3 & 0.39 & 0.14 & $\mathrm{E}$ & $\mathrm{S}$ & 1 and 2 \\
4 & Sept. 25 & $0624: 35$ & $0626: 30$ & n.a. & n.a. & 0.16 & 0.38 & $\mathrm{~L}$ & $\mathrm{~S}$ & 1 \\
5 & Sept. 25 & $0648: 00$ & $0654: 00$ & n.a. & n.a. & 0.32 & 0.41 & $\mathrm{E}$ & $\mathrm{S}$ & 1 \\
6 & Oct. 2 & $1538: 00$ & $1542: 00$ & n.a. & n.a. & 0.13 & 0.55 & $\mathrm{~L}$ & $\mathrm{U}$ & 2 \\
7 & Oct. 9 & $1310: 00$ & $1325: 00$ & 1.5 & 2.2 & 0.46 & 0.25 & $\mathrm{E}$ & $\mathrm{S}$ & 1 and 2 \\
8 & Oct. 17 & $1206: 00$ & $1226: 00$ & $\geq 1$ & $\geq 5$ & 0.12 & 0.49 & $\mathrm{~L}$ & $\mathrm{U}$ & 1 and 2 \\
9 & Nov. 12 & $0739: 00$ & $0751: 00$ & n.a. & n.a. & 0.38 & 0.11 & $\mathrm{E}$ & $\mathrm{D}$ & 1 \\
10 & Nov. 21 & $1012: 00$ & $1021: 00$ & 1.1 & 2.7 & 0.23 & 0.15 & $\mathrm{E}$ & $\mathrm{S}$ & 1 and 2 \\
11 & Nov. 21 & $1106: 00$ & $1116: 00$ & 1.1 & 13.8 & 0.16 & 0.38 & $\mathrm{~L}$ & $\mathrm{~S}$ & 1 and 2 \\
12 & Nov. 30 & $0710: 00$ & $0715: 00$ & n.a. & n.a. & 0.25 & 0.25 & $\mathrm{E}$ & $\mathrm{S}$ & 1 \\
13 & Nov. 30 & $0715: 00$ & $0721: 00$ & n.a. & n.a. & 0.60 & 0.16 & $\mathrm{E}$ & $\mathrm{D}$ & 1 \\
\hline
\end{tabular}

